\title{
Percepción del tiempo de los estudiantes chilenos en tareas de In- ternet en función de su procedencia: urbana o semi-rural
}

\section{Chilean students' time perception in Internet based-tasks accor- ding to the origin: urban or semi-rural}

\author{
Ramón Cladellas Pros ${ }^{1}$ \\ Universidad Autónoma de Barcelona, España \\ Luis Ramón Cárcamo Ulloa \\ Universidad Austral de Chile
}

(Recepción: Octubre 2009 - Aceptación: Diciembre 2009)

\begin{abstract}
Resumen
El objetivo de este trabajo es comprobar hasta que punto la procedencia de los sujetos (urbana vs semi-rural) incide en la estimación del tiempo en tareas realizadas por Internet. Un total de 120 estudiantes chilenos con una media de edad de 15,33 años, la mitad de procedencia urbana y la otra mitad de procedencia semirural, estimaron el tiempo que pensaban había transcurrido en la realización de una tarea de "búsqueda de información” en Google y una “tarea de diálogo mediado por mensajería instantánea” de Messenger durante un tiempo real de 490 segundos.

Los resultados muestran diferencias significativas en la tarea de diálogo mediado por mensajería instantánea. Los sujetos semi-rurales estiman un menor tiempo que los sujetos urbanos (521seg./651seg.). Estos resultados permiten establecer la consideración de la estimación del tiempo como barómetro cultural, además de plantear una serie de cuestiones aplicables al ámbito educativo que podrían ser resueltas en futuros estudios.

Palabras clave: percepción del tiempo, cultura, tarea de búsqueda de información, tarea de diálogo por
\end{abstract} mensajería, nuevas tecnologías

\begin{abstract}
The aim of this paper is to examine the extent to which the origin of subjects (semi-urban vs. rural) affects their time estimation on tasks performed on the Internet. A total of 120 Chilean students with an average of age of 15, 33 years - half of them of urban origin and the other half of semi-rural origin - estimated the time spent in performing a "seeking information" task on Google and a "IM-mediated dialogue" task on Messenger in real time (490 seconds).

The results show significant differences with regard to the IM-mediated dialogue task. The semi-rural subjects estimated a shorter time than the urban subjects (521sec./651sec.). These results reflect that time estimation can be considered a cultural barometer. Moreover, research findings raise a number of issues applicable to the educational environment, which constitute an interesting avenue for future research.

Key words: time perception, culture, seeking information task, IM-mediated dialogue task, new technologies
\end{abstract}

1 Correspondencia: Ramon Cladellas Pros. Departamento de Psicología Básica, Evolutiva y de la Educación, Universidad Autónoma de Barcelona, Edificio B. 08193. Bellaterra (Barcelona), España. Tel: +34935814531 Email: ramon.cladellas@ uab.es Fax: +34935813329.

Luis Ramón Cárcamo Ulloa. Facultad de Filosofía y Humanidades, Universidad Austral de Chile. Instituto de Comunicación Social, Campus Isla Teja, Valdivia, Chile. Email: luis.carcamo@gmail.com 


\section{Introducción}

El tiempo psicológico es un elemento fundamental en la adaptación del organismo al medio. En la actualidad una de las finalidades más recurrentes para justificar procesos de cambios del mundo analógico al digital se orienta hacia un mejor dominio del tiempo. Tareas que se desarrollaban en papel y lápiz buscan su rápida transformación a formatos de ordenador, sin medir muchas veces los efectos de esos cambios en los seres humanos. Para Castells (1999) la transformación del tiempo bajo el paradigma de la tecnología de la información, moldeado por las prácticas sociales, es uno de los cimientos de la nueva sociedad.

Los conceptos que los escolares antes buscaban en enciclopedias de papel, hoy los encuentran en Google, alternativa que les exige la habilidad extra de discriminar. El recorte de tiempo con la sustitución de memos por e-mail en las empresas o instituciones, constituyen otro ejemplo. Por otra parte, en el plano de las comunicaciones instantáneas, la mensajería o chat no es más rápida que el teléfono, pero sí tiene una condición de permanencia distinta, pues prácticamente los usuarios permanecen disponibles o semi-disponibles mientras están conectados a la red. Más allá de una posición unívoca respecto de lo favorable o perjudicial que resultan estos procesos transformadores de la vida cotidiana, es necesario investigar cuáles son esos efectos reales. La percepción del tiempo en las dos acciones, juntamente con el correo electrónico, que con mayor frecuencia se desarrollan sobre Internet: búsqueda de información y diálogo mediado por mensajería, puede servir como un barómetro cultural que permita explicar el cambio social que se está viviendo.

Los trabajos clásicos en percepción del tiempo: Fraisse (1973), Orsini (1971), Piaget (1971), Piaget y Meylan-Backs (1971) explican que los sujetos interactuando en distintas tareas, estiman el transcurso del tiempo de forma diferente. Fraisse (1973) y Piaget (1971) valoran en formas diversas el factor velocidad de ejecución de la tarea. Mientras para Piaget la percepción de la velocidad es fundamental para la percepción del tiempo; para Fraisse no lo es necesariamente y plantea que la percepción del tiempo debe entenderse como una síntesis compleja de factores, donde la velocidad es uno entre otros.

En los modelos de almacenamiento en memoria se considera que el número de estímulos que son codificados durante un periodo de tiempo influye en la estimación del mismo (Ornstein, 1969). No obstante, la evidencia sugiere que la magnitud de la duración recordada no depende tanto de la cantidad de información almacenada, sino del número de cambios que ocurren en un periodo de tiempo.

En trabajos más recientes, Pointer (1989) propuso un modelo donde el factor decisivo para la percepción del tiempo es el número de cambios. El cambio es el índice psicológico del paso del tiempo, así cuando el intervalo se divide en un mayor número de segmentaciones se asumen como nuevos cambios y esto produce un efecto de elongación del tiempo

Por su parte, Morales (2005) propone un modelo de tiempo psicológico en el estudiante, pensando en explicar los dos extremos del paso del tiempo en una sesión de estudio: la duración prolongada del tiempo y la compresión temporal. Este modelo considera tres dimensiones (alerta, carga de información y dominio de la tarea) y las direcciones extremas del paso del tiempo (compresión temporal y duración prolongada). De esta forma, la interacción entre las tres dimensiones determina el sentido de dirección del paso del tiempo comprimido o prolongado. El concepto de alerta considerado por Morales (2005) tiene un sentido similar al concepto clásico de atención, planteado en análisis de percepción del tiempo. En este sentido, Correa, Lupiañez y Tudela (2007), señalan que los procesos de atención y percepción del tiempo pueden interactuar de múltiples formas: en situaciones que implican una gran consciencia del paso del tiempo, tales como el aburrimiento, la impaciencia o incluso un estado de enfermedad (Bayés, 2002) el tiempo parece alargarse hasta la eternidad. En cambio, cuando nos encontramos muy involucrados en la realización de una actividad absorbente (o cuando nos hacemos mayores, v.g., Gambara, Botella y Gempp, 2002), a menudo tenemos la sensación de que el tiempo vuela. En última instancia, estos fenómenos sugieren que el grado de atención que prestamos al fluir del tiempo altera nuestra percepción de la duración. En otras palabras, la atención deforma nuestra percepción del tiempo. 
Gambara et al. (2002) hacen especial hincapié en la diferencia existente al estimar un intervalo de tiempo vacío y uno lleno. Un intervalo vacío, sería un intervalo sin actividad entre su inicio y su fin. En líneas generales, éstos investigadores encontraron que, ante tareas donde el sujeto es un mero observador, los tiempos parecen más extensos que cuando la actividad implica interacción directa.

Cárcamo, Cladellas y Estaún (2007) observaron diferencias significativas en la estimación del tiempo cuando se desarrolla una tarea espacial en formatos papel v/s ordenador. Los datos obtenidos demuestran que la estimación del tiempo es siempre menor cuando la tarea espacial es desarrollada en formato papel frente a la misma desarrollada en formato ordenador. Aunque estos resultados pueden parecer contradictorios con supuestos aspectos motivacionales que podrían subestimar el paso del tiempo, son totalmente congruentes con la explicación de que la sobreestimación del tiempo puede venir provocada por la mayor cantidad de diagramas espaciales realizados en ordenador. En coincidencia con los estudios de Pointer (1989) el mayor número de cambios en un tiempo determinado, cuando realizan la prueba en ordenador, produciría el efecto de prolongar la estimación del tiempo.

Perriault (2005) en sus estudios en dinámicas de construcción de conocimiento con videoconferencias interactivas plantea que en los procesos de estimación del tiempo intervienen aproximaciones de memoria, atención y contingencias propias de la actividad específica que se esté desarrollando. Entre las principales conclusiones aportadas se señala que en procesos funcionales basados en videoconferencias pueden percibirse como más breves las sesiones que regularmente duran 60 ó 90 minutos.

La ruralidad y la urbanidad como factores culturales en la percepción del tiempo de los sujetos tienen una lógica repetida en los estudios, tanto desde las vertientes más antropológicas como en los estudios más psicométricos. Hall (1989) cuenta varias experiencias en que comunidades no industrializadas perciben el tiempo más lentamente, sin premuras, dejando que cada cosa ocurra en su momento.

Estudios desarrollados por Choudhury y Gorman (1999) sobre la velocidad y consistencia de los procesos de tiempo de reacción investigados en adolescentes rurales de Guatemala muestran que el peso de esa relación es menor que la reportada por adolescentes urbanos de sociedades más industrializadas.

Las condiciones geográficas de Chile posibilitan la coexistencia de vivencias de tiempo muy distintas. No sólo ruralidad versus urbanidad, sino una serie de matices establecidos por clima, condiciones de luz y labores muy diversas.

Desde esta óptica podemos postular que existe un Chile de ritmos acelerados (Romero, 1991; Brito, 1992), que se corresponde con el área metropolitana de Santiago y un Chile provinciano de las pequeñas urbes (150.000 habitantes). Ciudades que ven pasar el tiempo con tranquilidad y a ritmos más bien lentos; que mantienen una fuerte relación con los sectores rurales aledaños, donde cada cosa tiene su tiempo y las urgencias no son parte del día a día.

En la actualidad se puede decir que en todos estos rincones de Chile, culturalmente distintos, en mayor o menor medida la tecnología está llegando y la percepción de ese cambio cultural también impacta de formas diversas (Cárcamo, 2008).

Teniendo en cuenta todo lo anterior, con este trabajo nos proponemos estudiar un aspecto novedoso, pues no existe literatura sobre el mismo al respecto, que consiste en comprobar si la procedencia cultural de los estudiantes chilenos incide en la estimación del tiempo en la realización de tareas por Internet (búsqueda de información y diálogo mediado por mensajería instantánea). Aún cuando el proyecto redes escolares tengan una muy buena cobertura en liceos (Enlaces, 2005) siempre estaremos ante sujetos que viven culturalmente el tiempo (Hall, 1989; Levi Strauss, 1972).

Hasta ahora los estudios desarrollados en Chile sobre percepción del cambio tecnológico se instalan en una perspectiva cuantitativa que evalúa cifras de accesibilidad documentadas sobre la encuesta nacional CASEN (Caracterización Socioeconómica Nacional), la variedad de usos (Godoy, 
2006) o las diferencias generacionales a la hora de percibir la sociedad digital (PNUD, 2006). La comparativa entre urbanidad y semi-ruralidad nos debería permitir explicar el impacto que tienen las tecnologías entre grupos más impactados (con mayor acceso a ellas) y grupos menos impactados o más apegados a ritmos de vida tradicionales (con menor acceso a las tecnologías).

\section{Método}

\section{Participantes}

La muestra está formada por 120 estudiantes, de los cuales 62 (51,7\%) son mujeres y 58 (48,3\%) hombres. La edad media era de 15,33 años. La mitad de la muestra de adolescentes urbanos estudiaba en liceos de Santiago de Chile. Por su parte, la muestra de adolescentes semi-rurales estudiaba en liceos de las poblaciones de Corral y Máfil, las comunas más pequeñas y semi-rurales de la Región de los Ríos.

Todos los sujetos, en el momento de la investigación, cursaban el segundo año de enseñanza secundaria y pertenecían socio-económicamente al grupo medio-bajo según las clasificaciones del SIMCE (Sistema de Medición de la Calidad de la Educación Chilena).

\section{Instrumentos}

- Cuestionario de búsqueda de información

Un primer cuestionario correspondiente a la tarea de búsqueda de información. Este cuestionario se compone de dos partes: una primera, con las instrucciones para buscar información relevante (lugar y fecha de nacimiento, principales obras y lugar y fecha de fallecimiento) de alguno/s de los seis personajes propuestos; y una segunda parte, donde el sujeto tenía que dejar constancia de lo encontrado y contestar una serie de preguntas, entre las que figuraba el tiempo que pensaba que había transcurrido durante la realización de la tarea.

- Cuestionario de diálogo mediado por mensajería instantánea

Este cuestionario se corresponde con la tarea de diálogo mediado por mensajería instantánea. Al igual que el anterior, este cuestionario se compone de dos partes: una primera con las instrucciones de la tarea a realizar; y una segunda con una hoja de respuestas, entre las que figuraba el tiempo que pensaban que había transcurrido desde el inicio de la tarea.

\section{Materiales}

Se emplearon ordenadores (Pentium IV 1.80GHz con 128 MB RAM) que se encontraban en el laboratorio de la RED Enlaces de los colegios, para que las condiciones experimentales fueran conocidas e iguales para todos los participantes. En todos los casos la conectividad a Internet resultaba estable, equivalentes a banda ancha de 256 kbts o similar.

Para efectos de las actividades experimentales el sistema operativo en todos los casos era Windows 2000 y los estudiantes realizaron la prueba de "búsqueda de información" en Google con un navegador Explorer 6.0 y la prueba de “diálogo mediado por mensajería instantánea” de Messenger 5.2

\section{Variables}

Tiempo estimado: corresponde al tiempo medido en segundos que el sujeto piensa que ha transcurrido entre el inicio y el final de la tarea.

Tipo de tarea: variable con dos categorías: tarea vertical-informacional (búsqueda de información) y tarea horizontal-dialógica (diálogo mediado por mensajería instantánea). En la primera se tiene que realizar una búsqueda de información por Internet a través del Google; y en la segunda se trata de mantener una conversación con otra persona vía chat. 
Contexto cultural: esta variable también tiene dos categorías: urbano y semi-rural. En el primer grupo los sujetos están escolarizados en liceos de Santiago de Chile y en el segundo grupo, nos encontramos con estudiantes de los pueblos de Corral y Máfil, las comunas más pequeñas y semirurales de la Región de los Ríos.

\section{Procedimiento}

En una primera fase pre-experimental o fase piloto se visitaron 8 colegios urbanos y 4 semirurales con el objetivo de analizar, revisar y superar posibles incidencias que se pudieran producir en la propia fase experimental, especialmente las referidas a control de variables o condiciones de los laboratorios informáticos.

De todas las variables estudiadas, se puso especial énfasis en dos de ellas: tiempo total disponible para realizar la tarea y frecuencia semanal de acceso a la misma. De la primera se pudo concluir que un tiempo de 8 minutos y 10 segundos garantizaba un tiempo completo de actividad; y respecto a la segunda, se verificó que todos los sujetos (urbanos y semi-rurales) tenían un acceso frecuente a las tecnologías, que en el caso de la búsqueda de información las frecuencias medias oscilaban de 3,2 días a la semana para los semi-rurales a 3,4 para los urbanos; y de 3,1 días a la semana para los semi-rurales a 4,2 para los urbanos en la prueba de mensajería.

En esta fase experimental participaron 120 sujetos que habían pasado previamente por la fase pre-experimental. Se descartaron nueve sujetos por usar alguna de las herramientas (buscadores de información o mensajería instantánea) con una frecuencia menor a una vez por semana.

El procedimiento para todos los sujetos era siempre el mismo. Tenían que realizar dos actividades sucesivas: una de "búsqueda de información” (Google) y otra de “diálogo mediado por mensajería instantánea” (MSN) durante un tiempo de 8 minutos y 10 segundos. Las sesiones experimentales se realizaban en grupos de seis personas en un laboratorio con ordenadores

Entre grupo y grupo se invertía el orden de ejecución de las tareas, a fin de evitar que el efecto orden de ejecución pudiera influir en los resultados.

La recogida de datos se realizó a través de los dos cuestionarios explicados en el apartado de instrumentos. Los investigadores, previamente entrenados para ello, se desplazaron a los diferentes liceos. Antes de comenzar, se explicaron las instrucciones de las tareas a realizar y se garantizó la confidencialidad de los datos aportados. La participación en el estudio fue totalmente voluntaria. El proceso se realizó durante los meses de Mayo y Junio.

\section{Resultados}

Las respuestas fueron sometidas a un análisis de varianza con alguna variable independiente intragrupo (GLM). En concreto, en el diseño se contempla una variable independiente intergrupo y una variable independiente intragrupo. Los cálculos se realizaron con el paquete estadístico SPSS 15.0.

En el conjunto de los resultados se constata como el tiempo estimado por los sujetos en cada una de las variables estudiadas sobrepasa el tiempo real de 490 segundos.

Un análisis entre los tipos de tarea manifiesta diferencias significativas $F(1,118)=15,69 p=0,000$. Tal y como se puede observar en la siguiente Tabla 1, el tiempo estimado en la tarea de búsqueda de información (688,72 ms.) es superior al de la tarea de mensajería (588,28 ms.) 
TABLA 1: Medias del tiempo estimado entre tareas

\begin{tabular}{|l|c|c|c|c|}
\hline & Media & N & Desviación Típica & $\begin{array}{c}\text { Significación } \\
\text { (Bilateral) }\end{array}$ \\
\hline Tiempo Búsqueda & 688,72 & 120 & 308,073 & 0,000 \\
\cline { 1 - 4 } Tiempo Mensajería & 586,28 & 120 & 275,646 & \\
\hline
\end{tabular}

En el análisis entre contextos culturales diferentes, no se observan diferencias significativas $\mathrm{F}(1,118)=4,40 \mathrm{p}=0,038$; si bien, la media de tiempo estimado $(686,14)$ por los sujetos de procedencia urbana es superior a la media de tiempo estimado $((588,86)$ por los sujetos de procedencia rural, tal y como se puede apreciar en la siguiente Tabla 2.

TABLA 2: Medias del tiempo estimado entre procedencias culturales

\begin{tabular}{|l|c|c|c|c|}
\hline & Media & N & Desviación Típica & $\begin{array}{c}\text { Significación } \\
\text { (Bilateral) }\end{array}$ \\
\hline Tiempo Urbano & 686,14 & 60 & 320,691 & \multirow{2}{*}{0,038} \\
\cline { 1 - 3 } Tiempo Semi-Rural & 588,86 & 60 & 249,828 & \\
\hline
\end{tabular}

Respecto a la interacción entre las variables tipo de tarea y contexto cultural, no se observan diferencias significativas $\mathrm{F}(1,118) \mathrm{p}=0,202$.

En un segundo análisis se efectúo una prueba estadística de muestras independientes que compara la estimación de tiempo entre procedencias culturales distintas para cada una de las tareas.

TABLA 3: Medias de Estimación del tiempo y procedencias culturales

\begin{tabular}{|l|l|c|c|c|c|}
\hline & \multicolumn{1}{|c|}{ Grupo } & N & Media & $\begin{array}{c}\text { Desviación } \\
\text { Típica }\end{array}$ & \multirow{2}{*}{ P } \\
\hline \multirow{2}{*}{$\begin{array}{l}\text { Tiempo Estimado para Búsqueda } \\
\text { de información }\end{array}$} & Urbanos & 60 & 721,22 & 323,004 & \multirow{2}{*}{0,249} \\
\cline { 2 - 5 } & Semi-Rurales & 60 & 656,22 & 291,456 & \\
\hline \multirow{2}{*}{$\begin{array}{l}\text { Tiempo Estimado para Dialogo por } \\
\text { Mensajería }\end{array}$} & Urbanos & 60 & 651,07 & 318,379 & \multirow{2}{*}{0,009} \\
\cline { 2 - 5 } & Semi-Rurales & 60 & 521,50 & 208,203 & \\
\hline
\end{tabular}

Tal y como se puede observar en la Tabla 3, se observan diferencias significativas en el tiempo estimado por los sujetos urbanos y semi-rurales en la prueba de diálogo por mensajería.

Para una mejor interpretación de los resultados, en el siguiente Gráfico 1 se pueden observar las medias de estimación en segundos para cada uno de los grupos estudiados. 
GRÁFICO 1: Representación de estimación del Tiempo y procedencia

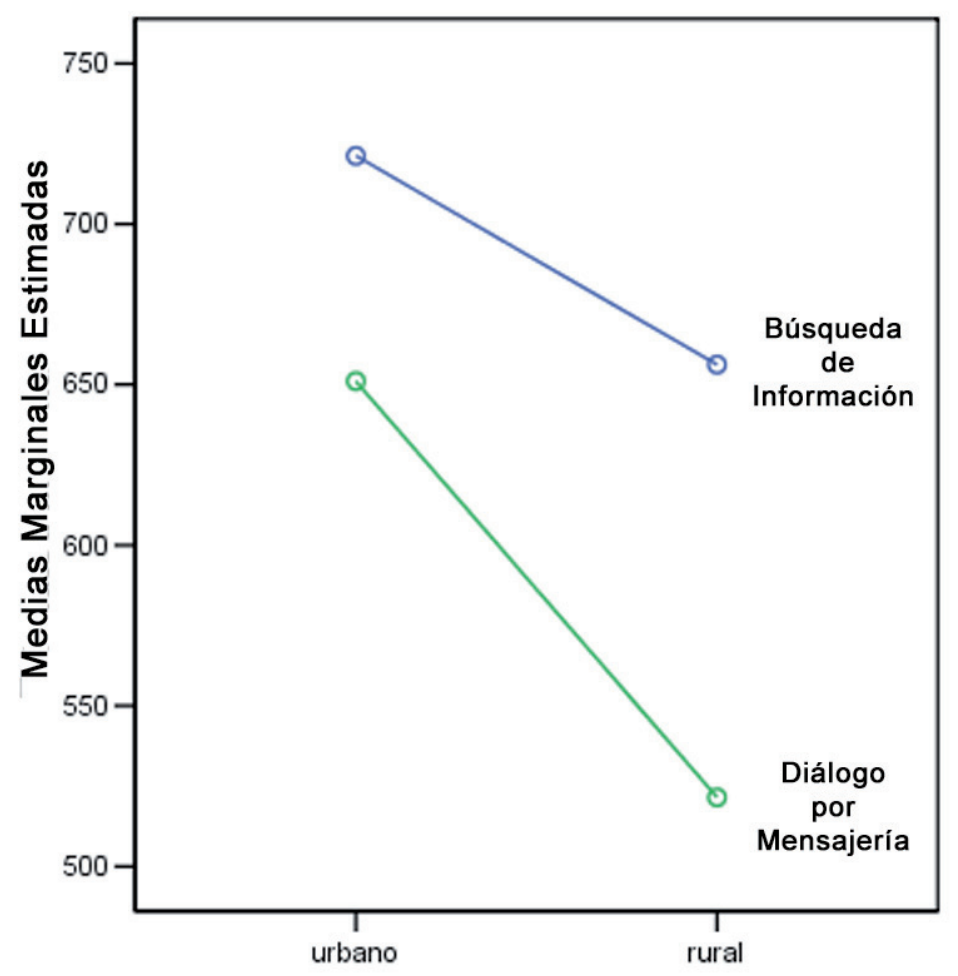

\section{Discusión y Conclusiones}

En términos generales y analizando descriptivamente los datos, los procesos de estimación del tiempo tienden a ser sobrestimados. El tiempo no pasa volando, al menos en los ocho minutos y diez segundos que comprenden las actividades experimentales desarrolladas.

Teniendo en cuenta que el objetivo principal de este trabajo ha sido analizar si el tiempo estimado en la realización de tareas realizadas por ordenador difiere según el contexto cultural, a tenor de los resultados, se puede concluir que el contexto cultural afecta en las tareas de diálogo mediado por mensajería instantánea, pero no así en las tares de búsqueda de información.

Las situaciones analizadas en este trabajo son procesos activos o también llamadas tareas de intervalos de tiempos llenos (Estaún, 1999; Gambara et al. 2002), donde el sujeto interactúa más o menos constantemente, ejecutando una "búsqueda de información” o “diálogo mediado por mensajería instantánea”.

Para las actividades de comunicación desarrolladas con tecnologías de la información y las comunicaciones, la memoria puede resultar un factor determinante dependiendo de la tarea que se esté ejecutando. Realizar síntesis biográficas implica un trabajo importante en la memoria. Esta tarea respondería al modelo de estimación de tiempo de Ornstein (1969), conocido como storatge-size model. En otras tareas, como la de diálogo mediado por mensajería instantánea, deberían tener mayor importancia el número de estímulos experimentados o change-segmentation como plantea Pointer (1989). Parece evidente que existe un factor cultural más dominante que el matemático, para explicar como los sujetos urbanos no estiman menos tiempo que los semi-rurales, a pesar que significativamente realizan más interacciones $(25,7 / 18,2)$, y por tanto reciben una mayor cantidad de estímulos. 
En este estudio se muestra, pues una diferenciación cultural que rompe el cálculo matemático del número de cambios percibidos y que se instala en el terreno de la valoración cultural de los estímulos. No son lo mismo 18,2 interacciones en Messenger para un sujeto rural que para un urbano. El sujeto urbano requiere un mayor número de estímulos para percibir un mismo periodo de tiempo.

TABLA 4. Número de Interacciones por procedencia cultural en la tarea de mensajería

\begin{tabular}{|l|c|c|l|c|}
\hline Procendencia & $\mathbf{N}^{\mathbf{0}}$ de interacciones & Tiempo estimado & & Media tiempo por intervalos \\
\hline Semi-Rurales & 18,2 & $=521,50 \mathrm{seg}$ & $\rightarrow$ & $28,6 \mathrm{seg}$ \\
\hline Urbanos & 25,7 & $=651,07 \mathrm{seg}$ & $\rightarrow$ & $25,3 \mathrm{seg}$ \\
\hline
\end{tabular}

De acuerdo con los datos presentados en la Tabla 4, se explicaría el modelo propuesto por Pointer (1989), donde el factor decisivo para la percepción del tiempo es el número de cambios. El cambio es el índice psicológico del paso del tiempo, así cuando el intervalo se divide en un mayor número de segmentaciones se asumen como nuevos cambios y esto provoca un efecto de elongación del tiempo.

En concordancia con los resultados presentados por Cárcamo et al. (2006) podemos decir que si en una tarea tediosa se producen muchos intervalos es normal que el tiempo se sobreestime, pero también puede resultar breve si la tarea resulta interesante. En tareas de comunicación la naturaleza vertical-informativa u horizontal-dialógica de las interacciones puede cobrar un valor especial. La tarea con una tendencia más interactiva y dialógica ofrece una menor estimación de tiempo pese a presentar un mayor dinamismo o número de intervalos.

Según el planteamiento realizado por Morales (2005) se podría entender que una menor estimación de tiempo por parte de los sujetos semi-rurales puede venir provocada por una menor frecuencia de acceso a la tecnología, que implicaría una mayor alerta en el momento de desarrollar la tarea y consecuentemente una compresión temporal.

Cabe señalar que los sujetos semi-rurales son los que ante la tarea de "diálogo mediado por mensajería instantánea” estiman el tiempo menor con una diferencia significativa de $\mathrm{p}=0,018$. Los sujetos semi-rurales estiman un tiempo de 521 segundos frente a 651 segundos estimados por los sujetos urbanos. En todos los casos se trata de lo que Morales (2005) define como duraciones prolongadas, pues la duración real es de 490 segundos.

Como plantea Reparaz (2001) las nuevas tecnologías están revolucionando el mundo de las comunicaciones, el receptor es quien selecciona la información que desea recibir y la forma como quiere obtenerla. Por ello, sería ideal que pudieran encontrarse en esta nueva etapa usos más aplicados y variados de las tecnologías de la información y las comunicaciones en el aula, y que para la búsqueda de nuevos procesos se tuvieran en cuenta la mayor motivación generada por los procesos de diálogo mediado por mensajería instantánea.

Chile establece culturalmente una diferencia entre los ritmos de Santiago como gran urbe industrializada y acelerada y los sujetos de provincia con una vinculación más rural. Evidentemente en la urbanidad influyen aspectos propios de la sociedad industrial moderna (Romero, 1991), con una idea de escasez de tiempo. Por su parte, en la ruralidad persiste una actividad temporal cíclica, propia de la actividad productiva campesina donde cada cosa tiene su tiempo. El cultivo, la crianza y la explotación de los recursos naturales responden a temporadas que no se pueden alterar. Las urbes como Santiago de Chile mantienen una cultura temporal compleja, debido a su gran extensión física, con un transporte que resulta precario y escaso para una población de más de cinco millones de habitantes. Esto sumado a una concepción dominante moderna del trabajo señalada por Brito (1992). En ese contexto los estudiantes de Santiago comienzan desde pequeños a vivir esas condiciones de la gran urbe. 
Desde la antropología, Levi-Strauss (1972) establece una clasificación binaria de las sociedades humanas, distingue entre 'sociedades frías' y ‘sociedades calientes'. Las primeras estarían identificadas con las primitivas o no occidentales, las segundas con aquellas que conocen la aceleración del tiempo, o sea con las de matriz europea. Puede ser que una sociedad más fría perciba una actividad dinámica y motivante como más breve pues el impacto del cambio resulta mayor. Ello concuerda con los resultados obtenidos en este trabajo, pues son los sujetos semi-rurales, menos habituados y consecuentemente más impactados ante una tecnología altamente motivante, los que perciben tiempos de estimación más breves.

Este tipo de estudios no está exento de limitaciones, pues como ya se apuntó en la introducción, Chile es un país con una gran variedad geográfica, climatológica y cultural. Estos factores convierten al territorio en una mina de pluralidad para la aplicación de estudios comparativos; si bien los prolongados desplazamientos y la escasa accesibilidad a algunos territorios encarecen y prolongan condiciones para un macroestudio.

Por todo lo anterior, las comparaciones presentadas en esta investigación tienen gran valor para los grupos investigados pero no necesariamente se puedan generalizar en otros grupos que conviven en la angosta y larguísima faja de tierra que comprende el territorio nacional.

Además, y como valor añadido, este trabajo predispone plantear una serie de preguntas, que podrían ser contestadas en futuros estudios.

- ¿ ¿Existe un umbral de tiempo a partir del cual los sujetos que trabajan con tecnologías comienzan a subestimar el periodo de trabajo?

- ¿ ¿Cómo varia ese umbral de tiempo en función de la naturaleza de las tareas ejecutadas en Internet?

- ¿ ¿Existen usos tecnológicos que se puedan asociar a factores culturales específicos?

Los nuevos desarrollos tecnológicos posibilitan poder estudiar nuevos procesos interactivos que se instalan y desinstalan no sólo de los computadores, sino también de los círculos sociales estableciendo formas de comportamiento colectivo. El cambio y la innovación se instalan en los usuarios de la red como una situación permanente.

Los Fotolog son un ejemplo claro de esas situaciones, hace cinco o seis años casi no existían, hoy se trata de una actividad puesta muy en práctica entre adolescentes y universitarios. Un segundo y no menos proyectivo fenómeno se instala a partir de la posibilidad de compartir videos en servidores de streeming como Youtube o googlevideo (Cárcamo, 2008).

Los resultados aportados en este trabajo pueden ayudar a las escuelas chilenas a propiciar una actividad docente proactiva, que sea capaz de observar las vivencias cotidianas de los estudiantes y servirse de ellas para incorporarlas creativamente a los procesos didácticos en la medida de lo posible.

\section{Referencias}

Bayes, R. (2002). Tiempo y enfermedad. Estudios de Psicología, 23 (1), 101-110

Brito, A. (1992) Percepción y valoración subjetiva del tiempo en estudiantes universitarios chilenos. Memoria para optar a título de Ingeniero Civil. Departamento de Ingeniería Civil, Facultad de ciencias físicas y matemáticas. Universidad de Chile.

Castells, M. (1999). La era de la información: economía, sociedad y cultura: La sociedad red Vol. I; Madrid, España: Alianza Editorial.

Cárcamo, L. (2008). Estimación de tiempo en estudiantes chilenos frente a tareas de búsqueda de información y comunicación desarrolladas con apoyo de Internet. Tesis Doctoral. Universidad Autónoma de Barcelona. Barcelona, España. 
Cárcamo, L., Cladellas, R., \& Estaún, S. (2007) Estimación de tiempo en estudiantes secundarios chilenos frente a una tarea espacial desarrollada en formatos de papel y ordenador. Revista Estudios Pedagógicos, 33 (2), 27-44

Choudhury, N. \& Gorman, K. (1999). The relationship between reaction time and psychometric intelligences in a rural Guatemalan adolescente population. International Journal of Psychology, 34 (4), 209-217

Correa, A., Lupiánez, J., \& Tudela, P. (2007). El tiempo: una dimensión clave en el estudio de la atención. Estudios de Psicología, 28 (1), 5-14.

Enlaces (2005). Cultura Digital en el sistema escolar chileno. Santiago de Chile: Enlaces-Ministerio de Educación.

Estaún, S. (1999). Percepción del Tiempo y Causalidad. En E. Munar, J. Roselló \& A. SánchezCabaco (Coords.), Atención y Percepción (pp.577-596). Madrid, España: Alianza.

Fraisse, P. (1973). Percepción y Estimación del Tiempo. En J.Piaget \& P. Fraisse (Comp.) Tratado de Psicología Experimental, Cap II, vol 6. Buenos Aires: Paidós.

Gambara, H., Botella, J., \& Gempp, R. (2002). Tiempo vacío y tiempo lleno. Un meta-análisis sobre los cambios en la percepción del tiempo en la Edad. Revista Estudios de Psicología, 23 (1), 87-100.

Godoy, S. (2006). Monitoreando el futuro digital: resultados encuesta WIP-Chile 2006 en Portal del World International Project, Chile.

Hall, E. (1989). El lenguaje Silencioso. Madrid, España: Alianza.

Lévi-Strauss, C. (1972) Estructuralismo y Ecología. Barcelona, España: Editorial Anagrama

Morales (2005) Tiempo psicológico en los estudiantes y carga de Información. Revista Ingenierías VIII.(27), 16-23

Ornstein, R. (1969). On the experience of time. Nueva York, USA: Penguin Books

Orsini, F. (1971). Contribución al estudio genético de la estimación del tiempo en función de la variación de las situaciones. En J. Piaget (Comp.), La Epistemología del Tiempo (pp. 162-190). Buenos Aires, Argentina: Editorial El Ateneo.

Perriault, J. (2005). Time and knowledge building, processes with interactive videoconferences. En A.N. Perret-Clemont (Ed.), Thinking Time, a Multidisciplinary perspective of time: Editorial Hogefre.

Piaget , J. (1971). La epistemología del tiempo. Buenos Aires, Argentina: El Ateneo.

Piaget, J. y Meylan-Backs, M. (1971). Comparación de operaciones temporales en relación con la velocidad y frecuencia. En J. Piaget y cols, (Eds.), La Epistemología del Tiempo (pp. 74-111). Buenos Aires, Argentina: Editorial El Ateneo.

PNUD (2006). Las nuevas tecnologías. Un salto al futuro. Programa de las Naciones Unidas para el Desarrollo, Chile.

Pointer W. D. (1989) Judging the Duratión of time Intervals: A process of remembering segments of experience. En I, Levin \& D. Zakay (Eds.), Time and Human Cognition. Netherland: Advanced In Psychology.

Romero, C. (1991). Factores que intervienen en la percepción y valoración del tiempo. Tesis para optar al grado de magíster en ingeniería industrial y título de ingeniero civil. 